\title{
Coronal Loop Mapping to Infer the Best Magnetic Field Models for Active Region Prominences
}

\author{
G. Allen Gary ${ }^{1}$, Qiang $\mathbf{H u}^{1}$ and Jong Kwan Lee ${ }^{2}$ \\ ${ }^{1}$ Center of Space Plasma and Aeronomic Research, The University of Alabama, \\ Huntsville, AL 35899, USA \\ email: gag0002@uah.edu, qh0002@uah.edu \\ ${ }^{2}$ Department of Computer Science, Bowling Green State University, \\ Bowling Green, OH 43403, USA \\ email: leej@bgsu.edu
}

\begin{abstract}
This article comments on the results of a new, rapid, and flexible manual method to map on-disk individual coronal loops of a two-dimensional EUV image into the three-dimensional coronal loops. The method by Gary, Hu, and Lee (2013) employs cubic Bézier splines to map coronal loops using only four free parameters per loop. A set of $2 \mathrm{D}$ splines for coronal loops is transformed to the best 3D pseudo-magnetic field lines for a particular coronal model. The results restrict the magnetic field models derived from extrapolations of magnetograms to those admissible and inadmissible via a fitness parameter. This method uses the minimization of the misalignment angles between the magnetic field model and the best set of $3 \mathrm{D}$ field lines that match a set of closed coronal loops. We comment on the implication of the fitness parameter in connection with the magnetic free energy and comment on extensions of our earlier work by considering the issues of employing open coronal loops or employing partial coronal loop.
\end{abstract}

Keywords. Magnetic fields, Models; Magnetic fields, Corona; Active Regions

\section{2D Cubic Bezier Splines, 3D Extensions, \& Misalignment Angles}

Gary, Hu, and Lee (2013) developed a rapid manual method to map individual coronal loops of a 2D EUV image as Bézier curves using only four points per loop. Their article outlined explicitly how the coronal loops can be employed in constraining competing magnetic field models by transforming 2D coronal loop images into 3D field lines. Their method uses the minimization of the misalignment angles between the magnetic field model and the best set of 3D field lines that match a set of closed coronal loops. The article considered only closed loops with given photospheric foot points; here we comment on the consequences of considering open loops and loop segments in the analysis.

The center of coronal loops is a locus of a point moving with one degree of freedom along the curve. One class of parametric curves is the Bézier curves. Cubic Bézier splines provide a curve that can fit most coronal loops with four number control points and hence provides a rapid matching process. Four $2 \mathrm{D}$ control points $\left[\mathbf{P}_{1}, \mathbf{P}_{2}, \mathbf{P}_{3}, \mathbf{P}_{4}\right]$ can be manipulated to provide a rapid fit to an EUV coronal loop image. In the previous study, the first and last control points were associated with the coronal-loop foot points. The four control points of the Bézier splines that are used to fit a coronal loop image are 2D, but these can be extended to 3D by the addition of z-components (Fig. 1). The non-zero z-components of the control points provide a 3D curve (psuedo field line) that will remain true to the projected coronal image. The first panel of Fig. 1 is for close loops and other two panels extend this concept to open field lines and a segment of a field line. 


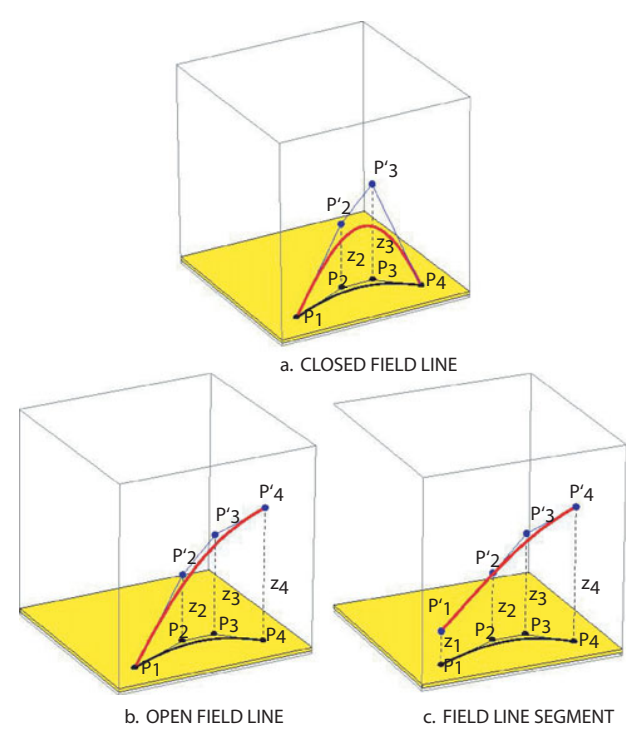

Figure 1. The extentsion of 2D cubic Bézier splines to $3 \mathrm{D}$ for closed, open, and partial loops. The thick lower (photospheric) line: a $2 \mathrm{D}$ cubic Bézier curve in the $z=0$ plane, defined by the four control points $\mathbf{P}_{i}\left(x_{i}, y_{i}\right)$. The 3D cubic Bézier curve (thick upper line) is obtained through the introduction of parameters: $z_{i}$. The two curves are related by having points lying at the same location in the image plane.

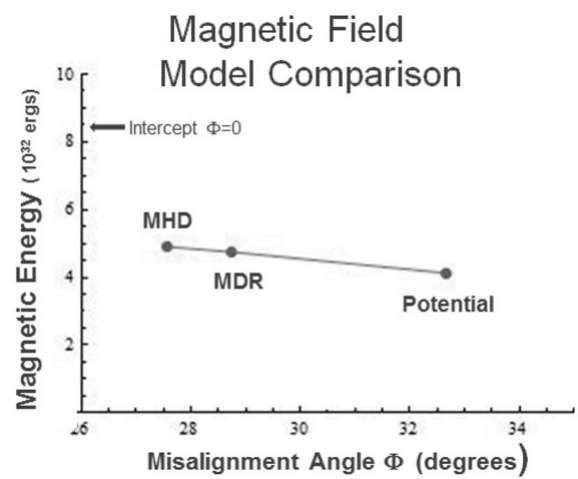

Figure 2. For the three magnetic-field models studied (potential, MDR, and MHD), the respective misalignment angles are $32.7^{\circ}, 28.8^{\circ}$, and $27.6^{\circ}$ for active region AR 11117 . The resulting magnetic-energy values in the volume are $4.12,4.47$ and $4.90 \times 10^{32}$ ergs. There is an almost inverse linear correspondence between the energy and the misalignment angle, with an extrapolated zero misalignment angle having an energy of about twice the potential energy.

\section{Concluson}

We can adjust the z-components of the 2,3 , or 4 control points to generate a best fit of the extended 3D spline to a magnetic field line. The requirement is the sum of the misalignment angles between the normalized tangents of the spline and the normalized magnetic field be a minimum. We have compared the average misalignment angle $[\Phi]$ to determine which magnetic field model best fits the mapped coronal images (see Gary, $\mathrm{Hu}$, \& Lee (2013) for details). Fig. 2 shows volume magnetic energy versus the misalignment angle. The results are for a potential field extrapolation compared with Wu's 3D time-dependent data-driven MHD solution, and, for comparison, the NLFFF minimum dissipative rate (MDR) model is included. This analysis assumed the identified loops were closed, i.e. both ends of the loop were coronal foot points at $z=0$. The MHD field is the best fit to the coronal loops, i.e. the lowest misalignment angles. It also has the highest magnetic energy. When assuming open field lines or segment of field lines, the minimization process becomes problematic, as a result of the misalignment angle having stronger inter-relationships near the photosphere.

\section{References}

Gary, G. A., Hu, Q., \& Lee, J. K. 2013, Solar Physics, August 2013 (on line), DOI: $10.1007 / \mathrm{s} 11207-013-0359-8$. 